\title{
Plantar fasciitis - to jab or to support? A systematic review of the current best evidence
}

This article was published in the following Dove Press journal:

Journal of Multidisciplinary Healthcare

24 May 2011

Number of times this article has been viewed

\author{
Hayley Uden' \\ Eva Boesch' \\ Saravana Kumar ${ }^{1,2}$ \\ 'Division of Health Sciences, \\ ${ }^{2}$ International Centre for Allied \\ Health Evidence, University of South \\ Australia, North Terrace, Adelaide, \\ South Australia, Australia
}

Background: Plantar fasciitis is a common condition routinely managed by podiatrists in the community and is widely treated conservatively. Two commonly used treatments for plantar fasciitis are customized functional foot orthoses and corticosteroid injections. While common to clinical practice, the evidence base underpinning these treatment strategies is unknown. Therefore, the aim of this systematic review was to assess the effectiveness and safety of customized functional foot orthoses and corticosteroid injections in the treatment of plantar fasciitis. Methods: A systematic literature search was conducted. Experimental studies, in English, from 1998 to 2010 were accepted for inclusion in this review. The PEDro quality assessment tool and the National Health and Medical Research Council's hierarchy of evidence were used to assess the quality of the included studies.

Results: Six randomized controlled trials which met the selection criteria were included in this review. Four reported on customized functional foot orthoses and 2 on corticosteroid injections. Current best available evidence highlights that both customized functional foot orthoses and corticosteroid injections can lead to a decrease in pain associated with plantar fasciitis. Additionally, customized functional foot orthoses may also provide an additional benefit in terms of increased functional ability in patients with plantar fasciitis. Corticosteroid injections may have side effects, especially pain (from the injection).

Conclusion: Both customized functional foot orthoses and corticosteroid injections can lead to reduction in pain associated with plantar fasciitis. While customized functional foot orthoses may increase the functional outcomes in patients with plantar fasciitis, corticosteroid injections may have side effects (especially pain as a result of the injection), which may limit its acceptability.

Keywords: plantar fasciitis, heel pain, orthotics, orthoses, cortisone injection, corticosteroid injection

\section{Introduction}

Evidence-based practice (EBP) is now a firmly entrenched philosophy for providing both safe and quality health care. Evidence based practice is defined as "the conscientious, explicit and judicious use of current best evidence in making decisions about the care of individual patients" " and is underpinned by 3 equally important concepts, namely, best research evidence, clinical expertise and patient values. ${ }^{1}$ While the importance of EBP is universally recognized, EBP in podiatry is still in its infancy. Podiatry's foray into EBP is shrouded by a lack of rigorous high quality evidence, limited research capability and/or opportunities for podiatrists, and barriers in accessing and implementing evidence into clinical practice. ${ }^{2,3}$ However, with increasing emphasis on EBP in health care, podiatrists too are required to ensure
Correspondence: Hayley Uden Division of Health Sciences, University of South Australia, North Terrace, Adelaide, South Australia, Australia Tel +6I 883022589

Fax +61883022766

Email hayley.uden@unisa.edu.au 
their practices are underpinned by current best research evidence.

Plantar fasciitis is a common condition routinely managed by many podiatrists in the community, as they are primary contact practitioners. Therefore, it is essential that podiatry management strategies for plantar fasciitis are underpinned by EBP principles to ensure that optimal outcomes are attained. Although most commonly described as an inflammatory condition (plantar fasci' ${ }^{\prime}$ itis), researchers have questioned the presence of inflammation in this condition. ${ }^{4,5}$ Plantar fasciitis is reported to commonly occur in runners and those who are overweight. ${ }^{6-9}$ Furthermore, research indicates that $10 \%$ of the general population will also experience this pathology at least once in their lifetime. ${ }^{6,7,10}$ The most commonly reported symptom of plantar fasciitis is described as "first-step pain", though plantar heel pain in general can be broadly associated with plantar fasciitis within the literature. . $^{6,911}$

The plantar fascia, also known as the plantar aponeurosis is a broad, flat, fibrous, tendon-like structure, which consists of noncontractile irregularly ordered collagen fibers with minimal elastic properties. ${ }^{12,13}$ It originates from behind the medial tubercle of the calcaneus and then divides distally in the area of the metatarsal heads into both its superficial and deep segments. The superficial fibers insert into the skin, whilst the deep segments attach to the transverse metatarsophalangeal ligaments and to the plantar surface of the proximal digits. ${ }^{13}$ The plantar fascia provides stability to the arch of the foot and aids in re-supination of the foot during propulsion. ${ }^{14,15}$

Conservative treatments remain popular for many patients with plantar fasciitis. A recent clinical practice guideline, recommends a number of conservative interventions in the management of plantar fasciitis. ${ }^{16}$ Among these conservative treatment options prefabricated foot orthoses (PFO), customized functional foot orthoses (CFO) and corticosteroid injections feature regularly (CSI)..$^{-11,16-22}$

Clinicians may be led to the use of PFO and CFO for the treatment of plantar fasciitis, in the belief that these provide a mechanical correction to the poor and/or altered biomechanics of the symptomatic foot, ultimately relieving the symptoms associated with plantar fasciitis. ${ }^{6}$ This treatment may be seen as having clinical benefit, as the clinician is trying to address the etiology of the condition, rather than simply "masking" the symptoms. Conversely, clinicians may look to use CSI as their treatment of choice as they are often considered a "quick fix", are relatively easy to administer, and time and resource efficient.
These conservative treatments are routinely used in clinical practice, and widely recommended, yet there is no evidence based consensus on the most effective treatment for plantar fasciitis. Emerging evidence suggests that both CSI and PFO should be considered as part of the "tier 1 treatment options", whilst CFO should be considered as part of the "tier 2 treatment options". However, the evidence base underpinning these recommendations could be considered to be low level and low quality. ${ }^{23}$ Despite this limited evidence base, in clinical practice, CFO and CSI remain popular, possibly driven by patient expectations and fee for service model. Confounding these issues are the known side effects, discomfort, and injection pain respectively for the use of CFO and CSI. ${ }^{4,5,23-26}$

Preliminary scoping search of the literature identified no study directly comparing CFO to CSI for the treatment of plantar fasciitis. In the absence of direct comparison between the two, literature which contained either of these as treatment options (with alternate comparators) was considered. Therefore, the primary aim of this systematic review was to assess the effectiveness of CFO and CSI in the management of plantar fasciitis. The secondary aim of this review was to report on the safety and, in particular, the side effects, associated with the use of these two interventions.

\section{Methods}

\section{Data sources}

Electronic databases were searched (Ovid [Medline], EMBASE, AMED, PubMed, SportDiscus, CINAHL, Cochrane Library, and AUSPORT) up to December 2010. The following search term combinations were used:

1. orthotic* or orthoses or functional foot orthotic* or functional foot orthoses or insert* or insole* AND plantar fasciitis or anterior medial heel pain or plantar heel pain or heel spur syndrome or painful heel syndrome or plantar aponeurosis

2. cortisone inject* or corticosteroid* inject* or steroid shot* or steroid injection AND plantar fasciitis or anterior medial heel pain or plantar heel pain or heel spur syndrome or painful heel syndrome or plantar aponeurosis. (*Indicates truncation)

\section{Study selection}

Only properly designed randomized control trials (RCT), which are considered to be level II in the hierarchy of evidence (National Health and Medical Research Council's [NHMRC] designation of levels of evidence), from the past decade, published as peer review journal articles 
were accepted for inclusion in this review. In the interests of accessing best evidence for this review, only the highest level of RCT (level II) were sought after for inclusion into this review. For the study to be considered a properly designed randomized control trial, the method of randomization needed to be both rigorous and adequately described. Alternate allocation and other means, such as date of birth and sequential, are not considered rigorous techniques of randomization. With rigorous randomization process, there is equal probability of participants being allocated to each and every treatment group. ${ }^{27}$ Inadequate randomization can lead to incorrect higher estimation of treatment effects, resulting in introduction of bias in the methodology. ${ }^{27}$ For this reason, inadequate randomization techniques, and studies without adequate descriptions of randomization methods, were not considered for inclusion into this review. The other limiters include English language publications and research in human subjects only.

Only subjects with a diagnosis of plantar fasciitis were included as the population group. Custom made functional foot orthoses needed to be a pair of in-shoe devices that were moulded or milled from impressions of the feet, whether by plaster cast or 3D laser scan. The fabrication then needed to be carried out according to practitioner-prescribed specifications. ${ }^{28}$ The CSI treatment had to involve an injection of a corticosteroid solution into the area of the plantar fascia.

Types of comparators included were, but not limited to: Achilles' tendon and plantar fascia stretching programs, extracorporeal shock wave therapy, manipulations of the foot and ankle, prefabricated foot orthoses, night splints (calf and/or plantar fascia), true "sham" foot orthotic devices and autologous blood injections.

Pain and function were chosen as the outcome measures of interest as these measures are routinely collected and reported in clinical practice.

Authors HU and EB reviewed all potential search "hits" following the results of the above literature search. The full text, the abstract, or the design methodology was used to ascertain the relevance of the article to this review. Any potential articles were set aside for further scrutiny and then from these, the final articles were selected.

\section{Methodological quality assessment}

The authors collaboratively assigned the chosen articles to the appropriate level of hierarchy with the NHMRC designation of level of evidence categories. ${ }^{29}$ PEDro quality assessment tool was applied to the included articles by both HU and EB independently. Any discrepancies in the assigned marks were addressed during discussions between $\mathrm{HU}$ and $\mathrm{EB}$ until agreement was found. The PEDro assessment tool contains 11 domains (10 included in the final score) which appraise the methodological rigor of randomized control trials and clinical control trials. ${ }^{30}$

\section{Data extraction}

The data were extracted independently by HU and EB using the same data extraction table. The data tables were then compared and any difference in the data collection was further scrutinized until resolution was found. Demographic data were collected pertaining to the subjects within the selected studies, as were the outcome measures utilized and the results of the within-group changes for each treatment arm of interest. Data relevant to the information utilized by the PEDro instrument were also collected. ${ }^{30}$

\section{Results \\ Search results}

Six properly designed RCTs (level II evidence) were retained for this systematic review. ${ }^{6,9,20,23,31,32}$ Seven pseudorandomized trials were identified from the literature but due to an inadequate randomization process or the absence of randomization description, were excluded from this review. ${ }^{8,11,17,19,33-35}$ A summation of both the included and excluded studies can be found in Table 1. Figure 1 provides an overview of the literature selection process.

Four studies investigated the effectiveness of CFO. ${ }^{6,9,20,32}$ The study by Roos and colleagues ${ }^{6}$ randomized a total of 43 subjects into a CFO group ( $\mathrm{n}=10$ at final), a night splint group ( $\mathrm{n}=15$ at final) or a combined night splint and CFO group ( $\mathrm{n}=13$ at final). Assessment measurements of pain and function were measured at baseline; $6,12,26$, and 52 weeks. Landorf and colleagues ${ }^{9}$ recruited a total of 136 participants initially and randomized them into 1 of 3 groups; a sham orthotic group ( $\mathrm{n}=43$ at final), a prefabricated orthotic group ( $n=43$ at final), or a CFO group ( $n=45$ at final). The outcomes of pain and function were measured at 3 and 12 months post intervention. The study by Dimou and colleagues ${ }^{20}$ randomly allocated 20 subjects into either group: 1 which received chiropractic adjustments of the foot and ankle twice a week for 4 weeks $(n=10)$ or group 2 which received a pair of CFO to wear for 8 weeks $(n=10)$. The outcome of pain was measured subjectively and objectively, using an Algometer, at baseline, day 15, day 29, and then at 1 month. Baldassin and colleagues ${ }^{32}$ initially randomized 142 participants to either a prefabricated orthoses group $(n=72)$ or a CFO group $(n=70)$. The outcome of pain was measured 
Table I Included and excluded randomized controlled trials

\begin{tabular}{|c|c|c|}
\hline Article & $\begin{array}{l}\text { Randomization } \\
\text { technique }\end{array}$ & $\begin{array}{l}\text { Included/ } \\
\text { excluded }\end{array}$ \\
\hline Lee and Ahmad ${ }^{31}$ & Computer generated & Included \\
\hline Roos et $\mathrm{al}^{6}$ & $\begin{array}{l}\text { Randomly ordered envelopes } \\
\text { within a box }\end{array}$ & Included \\
\hline Landorf et al ${ }^{9}$ & Computer generated & Included \\
\hline Porter and & Envelopes & Included \\
\hline \multicolumn{3}{|l|}{ Shadbolt ${ }^{23}$} \\
\hline Dimou et $\mathrm{al}^{20}$ & $\begin{array}{l}\text { Selection of a slip of randomly } \\
\text { collated paper labeled either } \\
\text { group I or group } 2\end{array}$ & Included \\
\hline Baldassin et $\mathrm{al}^{32}$ & Opaque sealed envelopes & Included \\
\hline Lynch et $\mathrm{al}^{8}$ & NR & Excluded \\
\hline Martin et al" & NR & Excluded \\
\hline Pfeffer et al ${ }^{17}$ & NR & Excluded \\
\hline Turlik et $\mathrm{al}^{19}$ & NR & Excluded \\
\hline Kalaci et $\mathrm{al}^{33}$ & Consecutive allocation & Excluded \\
\hline $\begin{array}{l}\text { Mulherin and } \\
\text { Price }^{34}\end{array}$ & Consecutive allocation & Excluded \\
\hline Yucel et $\mathrm{a}^{35}$ & NR & Excluded \\
\hline
\end{tabular}

Abbreviation: NR, not reported.

at baseline, 4 weeks, and 8 weeks. The primary pain outcome was a modified subscale of the Foot Function Index (FFI), whilst the secondary pain outcome was pain elicited by palpation of the medial calcaneal tuberosity. Data were complete at final analysis for 54 participants in the prefabricated group and 51 participants in the CFO group. No "intention to treat" approach was applied to statistical analysis.

The remaining 2 studies evaluated the effectiveness of CSI. ${ }^{23,31}$ Porter and Shadbolt ${ }^{23}$ recruited a total of 125 patients and randomized them into group A ( $\mathrm{n}=64$ heels), which received 1 injection of a corticosteroid and group B $(n=61$ heels $)$ which received electro hydraulic shock

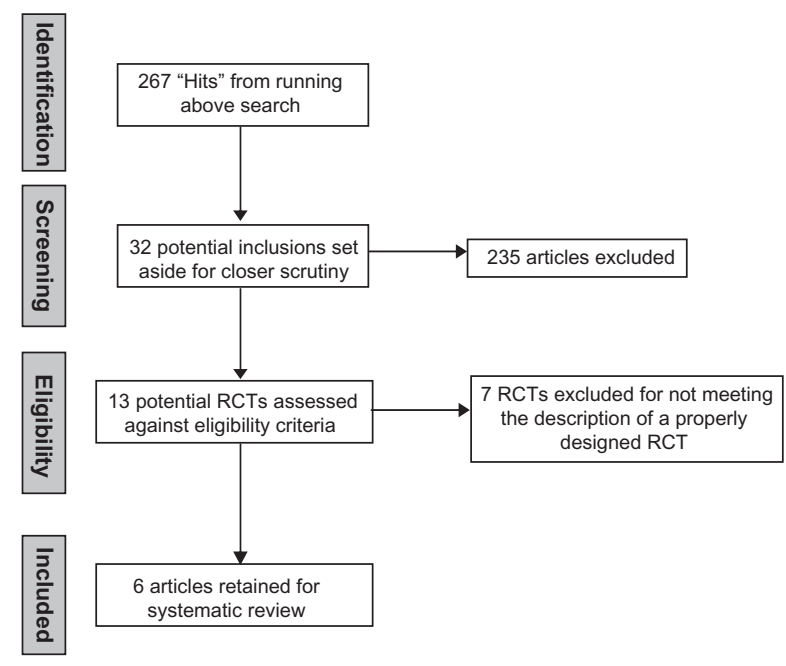

Figure I Literature selection process. Abbreviation: RCT, randomized controlled trial. wave therapy (ESWT). Both groups performed a standard stretching program for gastrocnemius, soleus, and plantar fascia. A third group C consisted of 19 "heels" that were eligible for the study but refused either a CSI or ESWT treatment and therefore only performed the stretching exercises. All patients were assessed for pain measures at baseline, 3 months, and 12 months. Lee and Ahmad ${ }^{31}$ randomized 64 participants into an autologous blood intralesional injection $(\mathrm{n}=30)$ or CSI treatment group $(\mathrm{n}=31)$. The outcome measure of pain was taken at baseline, 6 weeks, 3 months, and 6 months post treatment.

An overview of the psychometric properties for the outcome measures reported in the included studies is summarized in Table 2. A range of different outcome measures, including subjective and objective measures were reported. Subjective measures often related to pain severity and intensity (such as the Visual Analog Scale, Numeric Pain Rating Scale, and First Step Pain Scale) and also included measures of the impact of pain on function (Foot Health Status Questionnaire). Objective measures were primarily related to palpation findings (such as Tenderness Threshold). While psychometric properties of most of these outcome measures were recognized and reported, one study by Dimou and colleagues ${ }^{20}$ did not justify the psychometric properties of the First Step Pain Scale and the "Effect of Heel Pain on 3 Different Activities" form.

Roos et $\mathrm{al}^{6}$ reported no significant difference in pain scores (at any measurement points) between the 3 groups. When comparing the CFOs and the splint-only group, a clinically important difference in sport and recreation was observed at 26 weeks (assumed to be an increase in participation, but not stated). In addition, at 52 weeks the groups that were treated with CFOs reported a significantly higher pain reduction when compared with the splint alone group.

Landorf et $\mathrm{al}^{9}$ also reported a reduction in pain between study groups, but not a statistically significant reduction. Prefabricated foot orthoses and CFO were shown to cause a statistically significant improvement in function when compared with sham orthoses in the short term (3 months) but not in the long term (12 months). Dimou et $\mathrm{al}^{20}$ reported a significant difference for pain between the chiropractic treatment group and the CFO treatment group, with the chiropractic group being superior.

Baldassin and colleagues ${ }^{32}$ when comparing the effectiveness of low-cost prefabricated and customized foot orthoses, reported that both groups had similar outcomes. The findings from this study indicate that while there was a significant improvement in outcomes (both in pain and function) when 
Table 2 Outcome measures - psychometric properties

\begin{tabular}{|c|c|c|}
\hline Article & Outcome measures & Validity and reliability \\
\hline \multirow[t]{2}{*}{ Baldassin et $\mathrm{al}^{32}$} & VAS (modified pain scale from the FFI) & Validity and/or reliability reported \\
\hline & VAS (palpation at medical calcaneal tuberosity) & Validity and/or reliability not reported \\
\hline \multirow[t]{2}{*}{ Lee and Ahmad $^{31}$} & VAS & Validity and/or reliability reported [29] \\
\hline & Tenderness Threshold & Validity and/or reliability reported [30] \\
\hline Roos et $\mathrm{al}^{6}$ & Foot and ankle outcome score & Validity and/or reliability reported [6] \\
\hline Landorf et $\mathrm{al}^{9}$ & The Foot Health Status Questionnaire & Validity and/or reliability reported $[3 \mathrm{I}]$ \\
\hline \multirow[t]{2}{*}{ Porter and Shadbolt ${ }^{23}$} & VAS & Validity and/or reliability reported [29] \\
\hline & Tenderness Threshold & Validity and/or reliability reported [30] \\
\hline \multirow[t]{3}{*}{ Dimou et $\mathrm{al}^{20}$} & Numeric pain rating scale IOI (NRS-IOI) & Validity and/or reliability documented \\
\hline & First step pain scale & Validity and/or reliability not reported \\
\hline & "Effect of heel pain on 3 different activities form" & Validity and/or reliability not reported \\
\hline
\end{tabular}

Abbreviations: FFI, Foot Function Index; VAS, visual analog scale.

compared with baseline, there were no differences between the groups at 4 and 8 weeks post intervention. These findings indicate that low-cost prefabricated orthoses are at least as effective in reducing pain and improving function as customized orthotic devices.

The 2 studies which investigated the use of CSI demonstrated statistically significant reductions in pain with between group comparisons. ${ }^{23,31}$ However, Porter and Shadbolt reported that at 12 months the CSI and ESWT group had similar levels of average pain while the control group had significantly higher levels of pain. ${ }^{23}$ Similarly, Lee and Ahmad's study reported the corticosteroid group to show a significant reduction in pain on the visual analog scale at both 6 weeks and 3 months in comparison to the autologous blood group. ${ }^{31}$ However, this change was not significant at 6 months.

Table 3 provides a summary of within-group change from baseline to follow-up. There was a consistent finding of decreased pain across all studies, irrespective of the intervention. A clear trend was noticed across 4 of the studies with a reduction in pain. . $9,23,31$ This result was statistically significant within 3 of these 4 studies, Dimou et al being the notable exception with nonsignificant results. ${ }^{20}$ Function was reported in only 2 of the reviewed studies, which both contained a customized functional foot orthoses treatment arm. ${ }^{6,9}$ Both studies reported a statistical improvement of function at each of the points of assessment. It was of note that none of the studies which investigated corticosteroid injections used function as an outcome measure. ${ }^{23,31}$

Little information was provided about the safety of CFO's and corticosteroid injections in the included studies. Of the 4 studies $^{6,9,20,32}$ which investigated CFO, only one commented on the side effects of their use. ${ }^{6}$ Roos et al ${ }^{6}$ reported pressurerelated foot pain and tiredness of the foot in 3 of their subjects. Noncompliance with customized functional foot orthoses, a common clinical observation, was not reported in any of the studies. Both studies ${ }^{23,31}$ which investigated corticosteroid injections reported side effects as a result of their use. Porter and Shadbolt ${ }^{23}$ reported that all of the patients found the corticosteroid injection painful. Of the 64 heels injected, 8 cases required analgesia and/or ice application for postinjection pain. Similarly, in the study by Lee and Ahmad all of the patients found the corticosteroid injection painful. ${ }^{31}$ Of their cohort, $12.9 \%$ also experienced post-injection pain which required analgesia and/or ice application. ${ }^{31}$ The postinjection pain was said to have continued for a mean duration of 5 and 7 days respectively. ${ }^{23,31}$

The methodological rigor of the included articles was assessed using the PEDro instrument. ${ }^{29} \mathrm{~A}$ breakdown of the individual domain scores is provided in Appendix 1 (the higher the score out of 10, the less methodological bias within the study). Blinding of the subjects, the therapist, and/or the assessor were the domains on which most of the studies were flawed. In terms of blinding, only Baldassin et $\mathrm{al}^{132}$ and Landorf et $\mathrm{al}^{9}$ adequately fulfilled these criteria. Small sample sizes were also a characteristic of many of the included studies, with only three studies ${ }^{6,9,32}$ reporting power analyses to determine sample sizes.

\section{Discussion}

A systematic review, by its very nature, aims to evaluate best available research evidence, within a particular field of practice, with particular emphasis on rigor, precision, and generalizability. Within fields of practice where evidence is sparse, mixed and/or inconclusive, a systematic review of the current best available evidence provides useful information on current implications for practice and future research. The decision on whether to use CFO or CSI for the treatment of plantar fasciitis is one such area where the current best available evidence needs to be established. 
Table 3 Within-group change from baseline to follow-up

\begin{tabular}{|c|c|c|c|c|c|c|}
\hline \multirow[t]{2}{*}{ Study } & \multicolumn{2}{|c|}{ Intervention (CFO or CSI) } & \multicolumn{2}{|c|}{ Comparator I } & \multicolumn{2}{|c|}{ Comparator 2} \\
\hline & Pain & Function & Pain & Function & Pain & Function \\
\hline Baldassin et $\mathrm{a}^{32}$ & $\downarrow$ & $\uparrow$ & $\downarrow$ & $\uparrow$ & & \\
\hline Roos et $\mathrm{al}^{6}$ & $\downarrow$ & $\uparrow$ & $\downarrow$ & $\uparrow$ & $\downarrow$ & $\uparrow$ \\
\hline Landorf et al ${ }^{9}$ & $\downarrow$ & $\uparrow$ & $\downarrow$ & $\uparrow$ & $\downarrow$ & $\uparrow$ \\
\hline Dimou et $\mathrm{al}^{20, *}$ & $\downarrow$ & DNR & $\downarrow$ & DNR & & \\
\hline Porter and Shadbolt ${ }^{23}$ & $\downarrow$ & DNR & $\downarrow$ & DNR & $\downarrow$ & DNR \\
\hline Lee and $\mathrm{Ahmad}^{31, *}$ & $\downarrow$ & DNR & $\downarrow$ & DNR & & \\
\hline
\end{tabular}

Notes: *Studies which had only 2 groups.

Abbreviations: DNR, did not report; CFO, customized functional foot orthoses; CSI, corticosteroid injections.

The findings from this systematic review highlight several opportunities for reflection. Surprisingly, despite extensive literature interrogation, we were unable to find any high level evidence which compared effectiveness of CFOs with CSIs, despite these 2 treatments being widely promoted, recommended, and practiced in clinical practice.

The limited evidence base we did identify did pose unique challenges. The between-group results for the articles which contained a CFO treatment arm were mixed. ${ }^{6,920}$ The only statistically significant finding was that from the study by Landorf et al, where the CFO group and the prefabricated foot orthoses group were both superior to the sham group in respect to functional outcome in the short term (3 months). ${ }^{9}$ Similarly, the 2 articles which contained a CSI treatment $\operatorname{arm}^{23,31}$ demonstrated statistically significant improvements in pain in comparison with the other treatment arms but only in the short term.

The articles ${ }^{6,9,20,32}$ that investigated the effectiveness of customized functional foot orthoses were able to show a within-group reduction in pain at each and every assessment point. The study by Roos et $\mathrm{al}^{6}$ and Landorf et $\mathrm{al}^{9}$ contained the longest follow-up at 52 weeks post-intervention. This is in contrast to the Dimou et $\mathrm{al}^{20}$ study which evaluated only once at 8 weeks post-intervention. Baldassin et al $^{32}$ also reported similar findings with positive outcomes at 4 and 8 weeks post intervention.

All 4 studies scored well ( 8 points) in the PEDro appraisal, indicating only minor issues with methodological quality. The main drawback was a lack of blinding of subjects and therapists in Baldassin et al, ${ }^{32}$ Roos et al, ${ }^{6}$ and Dimou et al, ${ }^{20}$ which is to be expected given the nature of the intervention. The research by Dimou et $\mathrm{al}^{20}$ also utilized outcome measures with poor psychometric properties.

A within-group reduction in pain at each and every assessment point was also found in the 2 articles that assessed the efficacy of corticosteroid injections. ${ }^{23,31}$ A statistically significant reduction in pain was reported at each of these measurement points. Both studies evaluated outcomes at 52 weeks post-intervention, at which time a reduction in pain was still reported for the use of this treatment. However, both of these studies were constrained by similar methodological flaws (both with a PEDro score of 6). The threats to internal validity in these studies included failure to blind the subjects, therapist and assessor, and failure to instigate an intentionto-treat analysis.

Function was assessed in the studies by Landorf et al, ${ }^{9}$ Baldassin et al, ${ }^{32}$ and Roos et al. ${ }^{6}$ All these studies investigated the effectiveness of $\mathrm{CFO}$ and demonstrated a statistically significant increase in function at each of the measurement points. Adding to the strength of these results, all studies utilized outcome measures that had reported validity and/or reliability outcomes for their use within the literature. ${ }^{6,932}$ Considering that foot pathologies can have a profound effect on functional ability, it is interesting to note that not all studies attempted to measure function.

The risk factors for the use of CSI include plantar fascial rupture, hypoglycemia in diabetic patients, skin and fat-pad atrophy, and sepsis. ${ }^{4,24,25}$ However, this was not supported by evidence from the included studies. Neither of the studies involving a corticosteroid injection reported any such occurrences. ${ }^{23,31}$ Pain was however the primary risk factor reported consistently within these 2 studies. Universally, subjects in both the Porter and Shadbolt study ${ }^{23}$ and the study by Lee and $\mathrm{Ahmad}^{31}$ found the CSI painful. Not only was there injection pain but the associated post-injection pain was said to have continued for a mean duration of 5 and 7 days respectively. ${ }^{23,31}$ With regards to customized functional foot orthoses, only 1 study reported pain with the use of $\mathrm{CFO}{ }^{6}$

\section{Limitations of this review}

This systematic review, like any other research, has its limitations. The very nature of a systematic review ensures a very specific, targeted body of literature is identified, 
accessed, evaluated, and synthesized. As the review only included published, English language literature, the potential for publication and language bias should be acknowledged. While the authors made all attempts to identify and access all relevant studies, it is possible, due to differing terminologies, access to databases and journals, some may have been missed. There is also a paucity of evidence on cost-effectiveness and long term effectiveness of CFO and CSI for plantar fasciitis. Finally, this review is based on a modest body of evidence (6 RCTs) which were underpinned by several methodological flaws (such as small sample sizes). While the quantity and quality of primary research is beyond the reviewers' control, these limitations must be acknowledged when considering the findings from this review.

\section{Conclusion}

Currently there is limited research evidence which compares the effectiveness of CFO with CSI in the treatment of plantar fasciitis. This is an important evidence gap that needs to be addressed as plantar fasciitis is a commonly seen condition in clinical practice and clinicians are regularly confronted with needing to make informed decisions about CFO or CSI. Current best available evidence, with its inherent limitations, highlights that both CFO and CSI can lead to a decrease in pain associated with plantar fasciitis. Additionally, CFO may also provide an added benefit in terms of increased functional ability in patients with plantar fasciitis. In terms of harm and side effects, CSI may result in pain (from the injection). Clinicians using CFO and CSI should be aware of the limited evidence base and therefore routine use of CFO and CSI for plantar fasciitis should be carefully monitored and inform patient outcomes. This systematic review highlights current evidence gaps for two popular and increasingly accessible treatments for plantar fasciitis and emphasizes the importance of conducting ongoing high quality research in this area.

\section{Implications for clinicians}

As CFO and CSI seem to provide similar benefits for patients with plantar fasciitis, both these treatments could be considered as treatment options for plantar fasciitis. However, it is worthwhile to note that one of the side effects of CSI was pain as a result of the treatment procedure. While this may suggest an approach which favors CFO, which had minimal side effects reported in the literature, the cost of CFO may need to be considered. This scenario underscores the importance of collaborative decision making between the clinician and the patient, informed by best available evidence.

\section{Implications for future research}

Further research, such as RCTs, with sound methodological rigour, are required to investigate the effectiveness of $\mathrm{CFO}$ and CSI by directly comparing these 2 interventions. Future RCTs could also investigate the influence of the natural health course of plantar fasciitis by employing sham or a placebo group. As RCTs are time and resource intensive, it is likely that, in the short term at least, there will be an ongoing paucity of high quality literature. To address these gaps in the short term, well designed case studies can contribute to the evidence base for CFO and CSI. Future research should also focus on the cost effectiveness and long term effectiveness (including safety and side effects) of these two common treatment strategies.

\section{Authors' contributions}

$\mathrm{HU}$ and EB contributed to the conceptualization of the topic and the synthesis of all data. HU contributed to the development of the manuscript. SK contributed to the development, editing, and reviewing of the manuscript.

\section{Disclosure}

The authors declare that they have no competing interests.

\section{References}

1. Sackett DL, Rosenberg WMC, Muir Gray JA, et al. Evidence based medicine: what it is and what it isn't. BMJ. 1996;312:71-72.

2. Keenan AM, Redmond AC. Integrating research into the clinic - What evidence based practice means to the practicing Podiatrist. J Am Podiatr Med Assoc. 2002;92:115-122.

3. Hawke F, Burns J, Landorf KB. Evidence-based podiatric medicine - Importance of systematic reviews in clinical practice. J Am Podiatr Med Assoc. 2009;99:260-266.

4. Lemont H, Ammirarti KM, Usen N. Plantar fasciitis - A degenerative process (Fasciosis) without inflammation. J Am Podiatr Med Assoc. 2003;93:234-237.

5. Vohra PK, Kincaid BR, Japour CJ, et al. Ultrasonographic evaluation of plantar fascia bands - A retrospective study of 211 symptomatic feet. J Am Podiatr Med Assoc. 2002;92:444-449.

6. Roos EM, Engstrom M, Soderberg B. Foot orthoses for the treatment of plantar fasciitis. Foot Ankle Int. 2006;27:606-611.

7. Landorf KB, Keenan AM, Herbert RD. Effectiveness of different types of foot orthoses for the treatment of plantar fasciitis. J Am Podiatr Med Assoc. 2004;94:542-549.

8. Lynch DM, Goforth WP, Martin JE, et al. Conservative treatment of plantar fasciitis - A prospective study. J Am Podiatr Med Assoc. 1998;88:375-380.

9. Landorf KB, Keenan AM, Herbert RD. Effectiveness of foot orthoses to treat plantar fasciitis: a randomized trial. Arch Intern Med. 2006;166: 1305-1310.

10. Seligman DA, Dawson DR. Customized heel pads and soft orthotics to treat heel pain and plantar fasciitis. Arch Phys Med Rehabil. 2003;84: 1564-1567. 
11. Martin JE, Hosch JC, Goforth WP, et al. Mechanical treatment of plantar fasciitis: a prospective study. JAm Podiatr Med Assoc. 2001;91: $55-62$.

12. Tortora GJ, Grabowski SR. Principles of Anatomy and Physiology. 7th ed. New York: Biological Sciences Textbooks, Harper Collins College Publisher; 1992.

13. Marieb EN. Human Anatomy and Physiology. 5th ed, San Francisco: Benjamin Cummings; 2001

14. Michaud TC. Foot Orthoses and Other Forms of Conservative Foot Care. Massachusetts: Lippincott, Williams \& Wilkins; 1997.

15. Lisowski FP. A Guide to Dissection of The Human Body. 2nd ed. Singapore: World Scientific Publishing Company; 2004.

16. Thomas JL, Christensen JC, Kravitz SR, et al. The diagnosis and treatment of heel pain: a clinical practice guideline - revision 2010. J Foot Ankle Surg. 2010;49:1-19.

17. Pfeffer G, Bacchetti P, Deland J, et al. Comparison of custom and prefabricated orthoses in the initial treatment of proximal plantar fasciitis. Foot Ankle Int. 1999;20:214-221.

18. Rome K, Gray J, Stewart F, et al. Evaluating the clinical effectiveness and cost-effectiveness of foot orthoses in the treatment of plantar heel pain: a feasibility study. J Am Podiatr Med Assoc. 2004;94:229-238.

19. Turlik MA, Donatelli TJ, Veremis MG. A comparison of shoe inserts in relieving mechanical heel pain. The Foot. 1999;9:84-87.

20. Dimou ES, Brantingham JW, Wood T. Randomized, controlled trial (with blinded observer) of chiropractic manipulation and achilles stretching vs orthotics for the treatment of plantar fasciitis. Journal of the American Chiropractic Association. 2004;41:32-42.

21. Gross MT, Byers JM, Krafft JL, et al. The impact of custom semirigid foot orthotics on pain and disability for individuals with plantar fasciitis. J Orthop Sports Phys Ther. 2002;32:149-157.

22. Crawford $F$, Thomson CE. Interventions for treating plantar heel pain (Review). Cochrane Database Syst Rev. 2003;3:CD000416.

23. Porter MD, Shadbolt B. Intralesional corticosteroid injection versus extracorporeal shock wave therapy for plantar fasciopathy. Clin J Sport Med. 2005;15:119-124.

24. Speed CA. Corticosteroid injections in tendon lesions. BMJ. 2001;323: $382-386$.
25. Tsai WC, Scu CC, Chen CP, et al. Plantar fasciitis treated with local steroid injection: comparison between sonographic and palpation guidance. J Clin Ultrasound. 2006;34:12-16.

26. Valmassy RL. Clinical Biomechanics of the Lower Extremities. St. Louis: Mosby Year Book; 1996.

27. Soucacos PN, Johnson EO, Babis G. Randomised controlled trials in orthopaedic surgery and traumatology: Overview of parameters and pitfalls. Int J Care Injured. 2008;39:639-642.

28. Hawke F, Burns J, Radford JA, du Toit V. Custom-made foot orthoses for the treatment of foot pain. Cochrane Database Syst Rev. 2008;3: CD006801.

29. National Health and Medical Research Council [homepage on the Internet]. Australia: A Guide to the Development, Implementation and Evaluation of Clinical Practice Guidelines [updated 2010 July 20]. http://www.nhmrc.gov.au. Accessed March 18, 2011.

30. PEDro [home on the Internet]. Australia: Physiotherapy Evidence Database [updated 2011 March 7]. http://www.pedro.org.au/. Accessed March 18, 2011.

31. Lee TG, Ahmad TS. Intralesional autologous blood injection compared to corticosteroid injection for treatment of chronic plantar fasciitis: a prospective, randomized, controlled trial. Foot Ankle Int. 2007;28: 984-990.

32. Baldassin V, Gomes CR, Beraldo PS. Effectiveness of prefabricated and customized foot orthoses made from low - cost foam for no complication plantar fasciitis: A randomized controlled trial. Arch Phys Med Rehabil. 2009;90:701-706.

33. Kalaci A, Cakici H, Hapa O, Yanat AN, Dogramici Y, Sevinc TT. Treatment of plantar fasciitis using four different local injection modalities: A randomized prospective clinical trial. J Am Podiatr Med Assoc. 2009;90:108-113.

34. Mulherin D, Price M. Efficacy of tibial nerve block, local steroid injection or both in the treatment of plantar heel pain syndrome. Foot (Edinb). 2009;19:98-100.

35. Yucel I, Ozturan KE, Demiraran Y, Degirmenci E, Kaynak G. Comparison of high-dose extracorporeal shockwave therapy and intralesional corticosteroid injection in the treatment of plantar fasciitis. J Am Podiatr Med Assoc. 2010;100:105-110. 


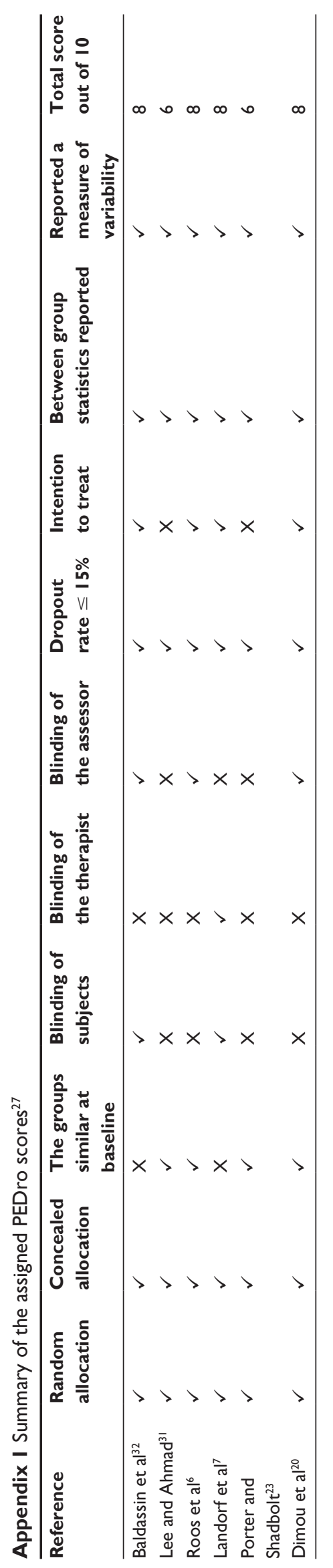




\section{Publish your work in this journal}

The Journal of Multidisciplinary Healthcare is an international, peerreviewed open-access journal that aims to represent and publish research in healthcare areas delivered by practitioners of different disciplines. This includes studies and reviews conducted by multidisciplinary teams as well as research which evaluates the results or conduct of such teams or

healthcare processes in general. The journal covers a wide range of areas and welcomes submission from practitioners at all levels, from all over the world. The manuscript management system is completely online and includes a very quick and fair peer-review system. Visit http://www.dovepress.com/testimonials.php to read real quotes from published authors.

Submit your manuscript here: http://www.dovepress.com/journal-of-multidisciplinary-healthcare-journal 